\title{
The integration of some applications as strategies in the teaching of FFL
}

\section{[L'integration de quelques applications comme strategies dans l'enseignement du FLE]}

\author{
Teute Blakqori
}

DOI: 10.18355/XL.2020.13.01.09

\begin{abstract}
In this paper, we will show the most used educational applications in addition to teaching literature and the French language at the University of Prishtina. More specifically, these are some applications that serve students and especially beginners. At our university, we sensitized our students to these new tools so that they learn French language and literature better by adapting them and practicing them in class. Just like books, applications, like highly interactive digital means, are necessary to learn and even serve as a good motivation for all the mechanisms leading to the acquisition of lessons learned in literary and linguistic lessons. Here we will see how a student feels at home by doing homework on the Internet and communicating with the teacher from a distance. With these new strategies, any student will learn more effectively and can be measured better his competence.
\end{abstract}

Key words: FFL teaching, application, strategy, interactive learning

\section{Résumé}

Dans ce travail, nous allons montrer les applications éducatives les plus utilisés en complément lors de l'enseignement de la langue et de la littérature française à l'Université de Prishtina. Plus spécifiquement, se dit de quelques applications qui rendent service aux étudiants et notamment aux étudiants débutants. Au sein de notre université, nous avons sensibilisé nos étudiants à ces nouveaux outils pour qu'ils apprennent mieux la langue et la littérature françaises en les pratiquants en classe. Tout comme les livres, les applications, comme des moyens digitales hautement interactives, sont nécessaires pour apprendre et servent même comme une bonne motivation à l'ensemble des mécanismes menant à l'acquisition des leçons données en cours littéraires et linguistiques. Nous allons voir comment un étudiant se sent à l'aise en rendant les devoirs sur Internet et en communiquant avec le professeur à distance. Grâce à ces nouvelles stratégies, tout étudiant apprendra plus efficacement et se fera mesurer mieux la compétence.

Mots-clés : enseignement du FLE, application, stratégie, apprentissage interactif

\section{Introduction}

Être ou devenir un enseignant stratégique en intégrant quelques applications numériques lors d'un cours FLE est devenu presque indispensable de nos jours. Ce

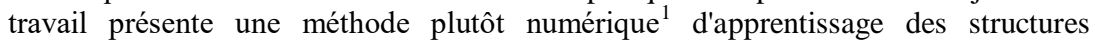
syntaxiques françaises qui convient aux personnes qui sont guidées par un professeur. Précisons tout d'abord qu'en classe de FLE ${ }^{2}$, l'utilisation des applications et de la méthodologie contemporaine a tellement évolué dispose de plusieurs approches de

\footnotetext{
${ }^{1}$ J'appelle méthode "numérique" toute astuce numérique (plateforme en ligne, application etc.) permettant à mieux enseigner et surtout à mieux faire comprendre les leçons dans les classes de FLE.

${ }^{2}$ Le terme de FLE est apparu en 1957 via les fameux travaux d'André Reboullet, enseignantchercheur français.
} 
l'enseignement du français qui ont déjà fait preuve de leur efficacité durant des années.

Aujourd'hui, le numérique prime sur le monde entier, et nous le voyons plutôt comme un outil fabuleux qui sert d'astuce stratégique permettant aussi bien aux professeur qu'aux apprenants d'échanger plus facilement, donnant plus de possibilité pour répondre massivement à une simple question posée par le professeur, principalement ou bien encore prioritairement, de faire un maximum de tâches en un très peu de temps. C'est justement l'appui sur ce pilier pédagogue qui accomplit par la suite le contenu de cette présentation. L'approche la plus récente du domaine de la méthodologie universelle demande à ce que les cours soient interactifs voyant l'apprenant comme le noyau de la réussite universitaire puisqu'on lui laisse la possibilité totale d'exprimer ses idées de manière naturelle en communiquant, en découvrant et en argumentant leurs idées ensemble. Selon notre expérience, nous avons découvert une augmentation de $50 \%$ de capacités de concentration et de mémorisation dans nos cours grâce aux techniques numériques utilisés comme stratégies efficaces pour apprendre, mémoriser et même enseigner de notre côté, en tant que professeurs, de manière beaucoup plus rapide sur tout type d'éléments éducatifs. Finalement, lors des examens de fin d'année, nous avons remarqué que les étudiants avaient gardé beaucoup mieux leurs informations mémorisées grâce aux applications utilisées. Tout cours universitaire muni du numérique renforce vraiment la motivation de l'étudiant car il pratiquera ses connaissances dans un format digital et visuel; cela l'aidera à mieux comprendre les cours et s'en souvenir plus longtemps. De plus, avec le numérique l'étudiant sera doté des compétences en numérique ce qui lui ouvrira plus de porte vers le marché du travail étant donné que posséder une aisance en compétences digitales est une priorité hautement demandée de nos jours. Il est donc temps à ce que nous tous, les enseignants, ayons aussi des compétences numériques pour que nos cours soient encore plus attractifs et plus intéressants.Notre travail consiste par une présentation générale, avec quelques stratégies et conseils pratiques pour faciliter le chemin vers la réussite d'un bon processus d'enseignement en classe de FLE.

\section{Le numérique en classe de FLE : quelques aspects et avantages généraux}

L'internet au service de l'enseignement de la langue et de la littérature françaises est indispensable dans une classe de FLE; ce qui n'existait pas à l'époque où la discipline nommée FLE faisant ses premiers pas dans les années soixante. De nos jours, la méthodologie dispose de nouveaux moyens d'enseignement, de communication accessible à tous et partout; quelles possibilités offertes par les technologies modernes? Et, principalement celles venant de l'Internet. Ce bon support pédagogique, depuis ses débuts, fascine les didacticiens spécialisés en méthodologie de l'enseignement car ils ont eu de meilleurs résultats avec le numérique. L'enseignement numérique (texte, son, image) permet l'intégration de l'approche actionnelle orientant l'apprenant vers une autonomie de l'apprentissage, l'échange entre les étudiants et le professeur pour rendre plus facile et spontané le processus de l'apprentissage.

Toutes les recherches théoriques, notamment celles des didacticiens spécialisés en méthodologie de l'enseignement, notamment: Reboullet de 1957 à 2010, Gadenne et Sotirakis 2003, Coianiz 2003, Desmet 2006, Aslim-Yetis 2009 Bihoué et Colliaux 2011, Cordina, Rambert et Oddou 2017 et Dominé 2017 nous font savoir que pour bien enseigner aujourd'hui, il nous faut intégrer les outils numérique dans nos cours. Parler du numérique en classe de FLE est avant tout décrire le résultat de tous les efforts que nous mettons lors de l'enseignement. D'ailleurs, apprendre une langue quelconque ou quelque chose dans une langue étrangère demande des efforts

XLinguae, Volume 13 Issue 1, January 2020, ISSN 1337-8384, eISSN 2453-711X 
particuliers à sa réalisation de façon fluide, indépendante et solide. Concernant l'évolution la méthodologie d'apprentissage à travers le temps, on distingue plusieurs courants d'apprentissage: la transmission, le béhaviorisme, le cognitivisme, le constructivisme, le socioconstructivisme, des courants donc allant d'une méthode primitive vers une méthode orienté principalement vers la discussion, des échanges sur place (en classe) ou à distance (par Internet). L'enseignement numérique peut être réalisable seulement dans une institution disposant les moyens nécessaires (logiciel, didacticiel, plates-formes d'apprentissage en ligne, tablette interactive ou tactile (IOS Android) smartphone, etc...)

Selon Lebrun, pour que les technologies catalysent réellement un renouveau pédagogique, la priorité serait mise sur l'importance de l'information, le support technique et le soutien pédagogique aux enseignants. Sans cela, les nouvelles technologies permettront au mieux de reproduire les anciennes pédagogies. Autrement dit, si les enseignants ne sont pas formés à ces technologies, dans bien des cas, ils risquent tout simplement de perpétuer les méthodes traditionnelles d'enseignement en utilisant un nouveau médium (Lebrun : 2004, p.17).

Avant de parler de notre expérience sur les applications utilisées en classe de FLE au Kosovo, pour un apprentissage plus facile et plus efficace, la connaissance d'un certain nombre de points généraux à ce processus (enseignement/apprentissage avec une application) est importante :

$\mathbf{1}^{\mathrm{er}}$ point : «On apprend beaucoup mieux ce qu'on entend et ce qu'on voit à la fois que ce qu'on entend. » L'inscription de signes linguistiques sur un support numérique renforce la concentration portée aux nouvelles informations.

$2^{\text {ème }}$ point: «On apprend beaucoup avec application car nous apprenons mieux ce qu'on a écrit/dit ou répondu nous-mêmes que ce qu'on n'a pas écrit/dit ou répondu nous-mêmes. » Cet appui a beaucoup été discuté ces dernières années dans le domaine de la "méthodologie d'apprentissage avec une application". Nous avons un bon article sur les applications d'apprentissage linguistique de $2019^{3}$ traitant de ce sujet et qui, d'ailleurs, mobilise aussi toutes les médias dans leur ensemble car c'est un thème constitué d'un grand nombre de possibilités diverses.

$\mathbf{3}^{\text {ème }}$ point : « Il est plus facile et agréable d'apprendre quelque chose sans se fatiguer trop physiquement, sans passer des heures et des heures à réunir tous les documents et porter tout ce matériel lourd dans une bibliothèque ou dans un bureau s'il le faut; une application peut éviter idéalement ce genre d'obstacle». Si nous devions posséder en format papier tous les documents nécessaires pour faire une dissertation un exercice d'argumentation autour d'une problématique quelconque organisée en plusieurs parties (introduction, développement, conclusion) cela serait une tâche longue, lourde et démotivante sans l'aide du numérique. Dans cet article, sous reviendrons à plusieurs reprises sur ce point.

\begin{abstract}
$4^{\text {ème }}$ point : «Attention à ne pas devenir accro aux applications quand-même. » Dit autrement, l'application aide d'une façon très efficace à l'enseignement/apprentissage. Mais, tous les didacticiens spécialisés en méthodologie de l'enseignement sont d'accord que cette méthode reste un complément. En fait, lire, s'entrainer, apprendre sans être dépendant à une méthode particulière demeure une réalité qui est très nécessaire pour réussir ses études; cela va de même pour réussir un bon enseignement et pouvoir à en faire le meilleur usage possible.
\end{abstract}

\footnotetext{
${ }^{3}$ Il s'agit de l'article « Les 7 meilleures applications d'apprentissage linguistique gratuites de 2019 - Apprendre langue », publié le 22 avril 2019, sur le site web suivant : http://www.eurolangues.org
} 
Ces points peuvent être un soutient crucial pour l'apprentissage du FLE. Toutefois, l'apprentissage d'une langue est vue comme un art complexe par plusieurs experts de ce domaine et notamment Lasserre qui disait «Il existe dans toute les langues, des choses traditionnelles que l'on ne peut pas expliquer et qui ne s'apprenne que par l'usage. C'est le cas du français, de l'emploi des prépositions, dans lequel reconnaissons-le - il y a souvent beaucoup d'arbitraire. (Lasserre: 1959, p.3)» (Cf. les exemples en (9) de ce travail). La méthode avec le numérique ${ }^{4}$ et la plus répandue est celle qui a envahi le monde, elle est très populaire et même si elle reste un complément, nous, en tant que enseignants francophones, nous avons vraiment besoin de son utilisation et de ses fonctionnalités.

\section{Le numérique en classe universitaire de FLE à Prishtina}

La nouveauté portée par cet article est liée au fait que nous avons mené des expériences qui démontrent que lorsqu'on doit apprendre une leçon, un peu complexe mais nouvelle, il est important d'avoir toujours à portée de main une application, noter ce que nous voulons retenir, les mots nouveaux. Les professeurs peuvent l'utiliser pour mieux expliquer les leçons ou les faire mieux comprendre par tous les étudiants. Nous savons tous que les premières informations mémorisées sont les plus solides et restent plus longtemps dans notre mémoire. Commencer avec des applications pour apprendre les informations les plus difficiles à retenir en premier est une très bonne stratégie. Nous avons voulu améliorer la méthodologie de l'enseignement avec nos étudiants. Après avoir identifié et géré les causes des objectifs essentiels, nous avons tenté d'améliorer la satisfaction et l'engagement de nos étudiants en FLE. Notre objectif était d'enseigner en passant moins de temps à analyser pour avoir plus de temps à agir en classe grâce aux moyens numériques, les applications. La technologie numérique évite le travail fatiguant (prendre des notes) et aide les apprenants à atteindre leurs objectifs plus vite et de façons satisfaisantes. De nos jours, nous avons une génération digitale, c'est pourquoi nous ne pouvons plus se contenter de donner seulement du contenu aux étudiants. En mettant en pratique les moyens digitaux, nous avons pu franchir le pas vers une meilleure réussite universitaire. D'abords nous avons sélectionné les outils digitaux indispensables à télécharger sur nos appareils (smartphones, tablettes, ordinateurs etc..) avant de commencer l'enseignement avec les applications. Ainsi, avec nos étudiants, d'abord, nous nous sommes donné les moyens d'obtenir les connaissances digitales et après nous avons utilisé des outils numériques tels que :

$\begin{array}{ll}\text { - } & \text { Google Classroom } \\ \text { - } & \text { Padlet } \\ \text { - } & \text { Kahoot }\end{array}$

Google classroom est une plate-forme d'apprentissage gratuite, dédiée aux établissements scolaires pour simplifier la création et la diffusion de cours et d'exercices de façon numérique. Google Classroom ${ }^{5}$ offre aux enseignants des outils

\footnotetext{
${ }^{4}$ Nous disposons sur Internet d'un mémoire de Master fait sur L'acquisition du français à l'usage d'applications mobiles: un état de question. 2014 par Stijn Haelewyck. C'est une recherche faite sur ce même thème laissent entendre que même la société moderne d'aujourd'hui est entièrement imprégnée de technologie mobile connaissant un essor considérable et pas seulement l'enseignement/l'apprentissage.

${ }^{5}$ Pour tout enseignant, il est important d'avoir une bonne aisance dans la pratique de cet outil informatique et posséder une véritable familiarité avec la culture de Classroom (https://classroom.google.com) afin que tout cours de FLE avec ce numérique soit réalisable.
}

XLinguae, Volume 13 Issue 1, January 2020, ISSN 1337-8384, eISSN 2453-711X 
pédagogiques permettant de regrouper tous les cours pour chaque année universitaire devenant ainsi un espace et une classe virtuelle. Plusieurs informations pratiques, comme les consultations avec les professeurs en direct ont également été intégrées. Classroom, a une mise en page virtuellement très efficace et visuellement attrayantes mais n'offre pas d'outils de recherche ou les matières étudiées de façon automatique mais sa fonctionnalité de classe virtuelle est tout à fait correcte. Cependant, le professeur et les étudiants peuvent créer une classe de travail pour suivre le cours entier et favoriser l'interaction. C'est une plateforme gratuite permettant la maitrise parfaite de plusieurs applications en même temps. C'est donc un outil éducatif permettant aux enseignants d'échanger plus facilement avec leurs étudiants. On utilise ce service gratuit pour évaluer, observer et partager avec les étudiants. Ceux-ci auront en face d'eux l'écran, ils peuvent voir les documents publiés par leurs professeurs mais aussi les devoirs soumises. Il est nécessaire que la salle soit équipée avec des hautparleurs, au cas où il aura des liens ou le son est incorporé (pour le cas d'une dictée). Les enseignants sont un espace, sous forme d'une page pour créer leurs cours, quant aux étudiants, ils doivent par la suite rejoindre le cours avec le code fourni par le professeur précédemment. Tout se crée vite, facilement et automatiquement, il suffit de suivre les instructions jusqu'à la réalisation finale et en faire les demandes nécessaires: pour obtenir un code d'accès, joindre le cours, soumettre des devoirs, télécharger les cours que l'on a manqué pour une raison ou pour une autre etc...

L'objectif est avant tout de :

connaître différents outils de collaboration en ligne, d'évaluation en ligne et de création en ligne.

- maîtriser les aspects techniques et les fonctionnalités de la plateforme Google Classroom.

- Adapter et éditer des documents vidéo et audio.

- identifier les différentes étapes conduisant à la création d'un enseignement hybride ou à distance.

- caractériser les différents enjeux et conditionnalités des outils numériques.

- identifier les contraintes liées à l'intégration des outils numériques

- comprendre différents modèles pédagogiques appliqués en intégrant les TICE.

- réfléchir sur comment utiliser ces outils dans une situation d'apprentissage à distance.

En général, les apprenants montrent un vif intérêt pour tous les modules utilisant classroom, les jugeant très utiles pour la préparation de la conception d'un cours en ligne. Ils apprécient la méthodologie participative et la possibilité de se consacrer plus à la pratique des notions abordées.

\section{Applications pour les cours en Littérature Française}

Concernant les cours de littérature offerts dans le département de FLE à l'Université de Prishtina, nous disposons d'une multiplicité de sujets et types de textes littéraires, disponibles en langue français évidemment, mais pas disponible en format papier pour tout le monde. Ayant un intérêt professionnel dans la littérature française, les cours de littérature sont adaptés aux étudiants de tous les niveaux à temps plein. Ils sont repartis sur plusieurs semestres; du début jusqu'à la réussite du diplôme, en passant par tous les mouvements littéraires français: Moyen Âge/ Renaissance, Humanisme (XVIème), Baroque (XVIIème), Romantisme /Réalisme 
/Symbolisme/Surréalisme (XIXème) et littérature contemporaine. Pour ces cours de littérature, les étudiants doivent lire un certain nombre de textes, des pensées critiques, mais aussi ils doivent présenter leurs idées, faires des interprétations littéraires et des essais. Il est vrai que parler de toute la littérature française est une mission presque impossible car c'est un champ très vaste anticipant sur de grandes synthèses contemporaines. C'est pour cette raison que l'intégration des applications numériques en classe de FLE est très importante. Dans le cas contraire, les cours de littérature dureraient quelques heures par semaine et les étudiants de notre département ne pourraient pas avoir accès facilement et avec un peu de moyen financiers à cet immense et riche ensemble des œuvres écrites en français. Grâce aux applications et tous les autres moyens numériques que nous disposons aujourd'hui, la littérature française appartient à tout le monde maintenant, et particulièrement à tous ceux qui veulent l'étudier et percevoir ses significations, ses messages magistraux. Au sein de notre département, nous avons testé essentiellement les trois outils ci-hauts mentionnés, illustrés ici par nos exemples :
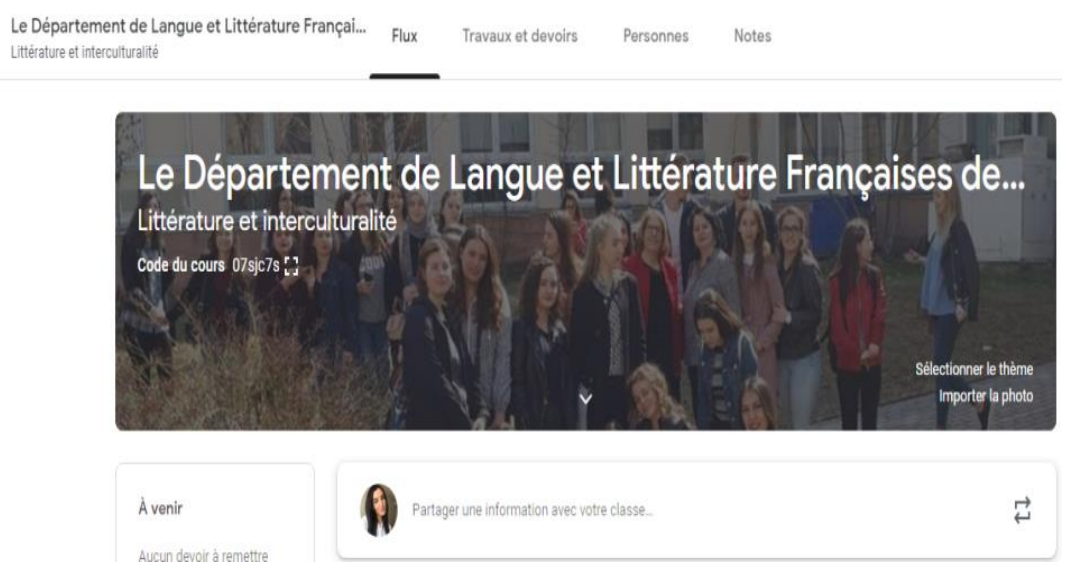

Figure 1: La classe virtuelle «Classroom» crée dans le département de FLE à Prishtina

Padlet est un outil crée en 2016 qui permet de créer un mur virtuel et partager en groupe des documents, exposés, commentaires. Padlet est chargé de réaliser des taches évaluées sur un mur blanc personnel faites par l'enseignant y compris les rendez-vous en ligne. Sur ce mur nous pouvons déposer des notes, des liens, des images, des sons, des vidéos, des fichiers Word ou Excel, des PDF. Les étudiants peuvent compléter ce mur virtuel avec des textes, images, enregistrements sonores, et en tant que professeur, nous pourrons décider de tout sur le mur : la sauvegarde ou l'effacement d'un élément quelconque y compris pour la création de son compte ${ }^{6}$. L'étudiant a accès au mur padlet toujours et peut déposer un travail ou des commentaires en dehors de la classe, ce qui facilite son travail en autonomie. Padlet permet de retrouver facilement tous les liens disponibles pour les leçons d'un cours particulier; c'est un travail interactif en linge. Grâce à ses fonctionnalités, l'application est capable de regrouper collectivement ou individuellement les travaux

\footnotetext{
${ }^{6}$ Le professeur doit se connecter, se rendre à cette adresse : https://padlet.com/auth/login et y créer un compte.
}

XLinguae, Volume 13 Issue 1, January 2020, ISSN 1337-8384, eISSN 2453-711X 
de recherches documentaires en rassemblant les résultats sur un seul Padlet, un seul tableau.

Avec ce bon moyen stratégique, nous pouvons faire plusieurs projets de recherche et réunir un grand nombre d'étudiants de quelques simples claquements de doigt sur l'écran, effectués sur un smartphone ou sur une tablette.

L'avantage du mur est de mettre en évidence l'importance de l'investissement de chaque étudiant et sa répercussion sur la qualité du travail collectif final. Voici un exemple fait par les étudiants du département de français sur L'apprentissage de la littérature française aux lycées du Kosovo.

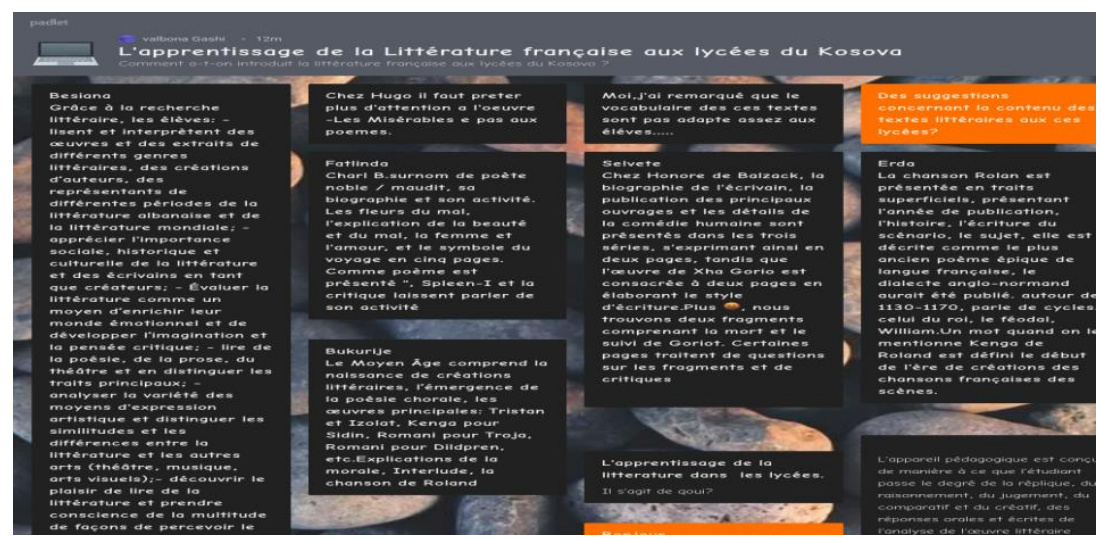

Figure 2 : Padlet réalisé avec les étudiants du département de français.

Quelques parties des exposés sont mises sur le mur de ce Padlet par des étudiants qui ont travaillé au module L'enseignement de la littérature française. Les consignes ont été donnés au début du semestre et les étudiants ont du choisir un auteur français selon le programme du Ministère de l'éducation du Kosovo. Comme support pédagogique, ils se sont servis du livre scolaire sur La littérature mondiale de l'auteur, Ardian Marashi.

La structure de ce mur Padlet reflète l'objectif didactique avec la consigne qui a été donnée par le professeur et les interactions après chaque présentation.

Kahoot : est un outil en ligne, et qui permet de réaliser très simplement des quiz pour animer un cours présentiel : le quiz sera lancé à partir d'un ordinateur et avec le support d'un écran/projecteur dans la classe. Les questions possèdent aussi des images pour aider les étudiants à mieux comprendre la question et en même temps pour mieux mémoriser. Les outils nécessaires sont le projecteur, réseau d'internet, des ordinateurs, tablettes, Ordinateur ils peuvent utiliser aussi leurs smartphones. Il est préférable d'être appliqué dans les 20 dernières minutes pour savoir si les étudiants ont bien acquis le contenu donné durant le cours. Le professeur, après avoir ouvert son compte Kahoot, il lui est nécessaire de créer le quiz kahoot en posant des questions nécessaires qui donneront une vive réaction et saura si les étudiants ont bien acquis les connaissances.

Par la suite, nous verrons comment nous avons utilisé en classe de FLE Padlet et Kahoot comme d'excellents moyens pour exposer nos idées à tous sans que personne soit mis à l'écart sous prétexte qu'il n'y avait pas le temps ou n'avait pas pu être présent en classe. 
Le questionnaire a donc été distribué de façon numérique via Kahoot, il est construit à base de textes déjà étudiés. Nous avons pris l'exemple de l'œuvre de Victor Hugo Les Misérables.

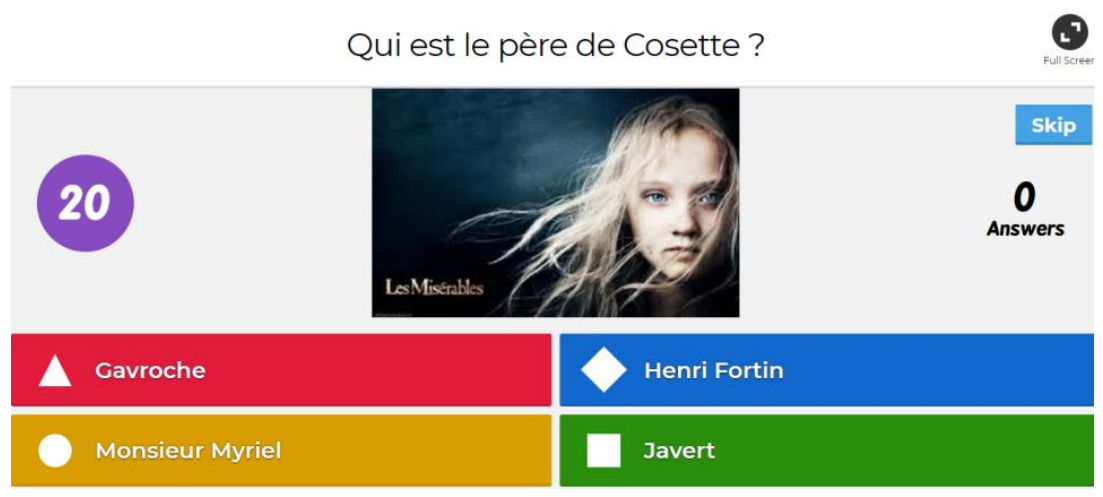

Figure 3 : Kahoot réalisé avec les étudiants du département de français.

Ce quiz se base uniquement sur des conseils méthodologiques et propose de mettre en place une stratégie pour mieux faciliter l'apprentissage en leur permettant l'obtention des bases solides. Il se pratique seul ou à plusieurs, Cela dépend de l'enseignant, c'est à lui de préciser les procédures élaborées; il a la possibilité de créer un Padlet sous forme de questionnaire simple ou à choix multiples.

\section{Applications pour les cours en Linguistique Française}

Les mêmes applications sont conçues uniquement pour les apprenants en FLE, avec des outils de contrôle professionnel pour que le professeur les accompagne convenablement. Comme partout ailleurs pour l'enseignement du FLE, au Kosovo aussi, l'apprentissage de la langue française par nos étudiants est axé sur les quatre critères d'appréciation : lecture, compréhension, expressions orale et écrite. Nous utilisons les mêmes applications pour les cours de linguistique française aussi, ils sont repartis en cours magistraux durant cinq années d'étude (Licence et Master) dans le département de langue et littérature française à Prishtina. Notre programme FLE propose tous les aspects linguistiques du français; en commençant par la phonologie, la grammaire (les groupes nominaux, verbaux, et phrastiques). L'utilisation des outils numériques est très importante et nécessaire pour éviter les erreurs faites par apprenti débutant albanais rencontrant généralement des difficultés associées à l'apprentissage $\mathrm{du}$ français langue seconde.

En phonologie -la difficulté la plus pertinente touche la prononciation, notamment celle des quatre voyelles nasales françaises manquantes dans notre langue. En albanais, l'acquisition de ces voyelles nécessite des efforts supplémentaires et des répétitions multiples par nos étudiants. Padlet, nous aide beaucoup dans la solution de ces problèmes. Les étudiants nous ont surpris par leur vitesse d'apprentissage de la prononciation et l'écriture; ils nous ont dit que grâce aux liens que nous, les professeurs, avions mis en linge via Padlet, sur notre classroom ; ils ont pu s'entrainer jusqu'à la perfection, à la maison et partout d'ailleurs puisqu'une application rend possible cette fonctionnalité libre. La prononciation française de façon correcte est

XLinguae, Volume 13 Issue 1, January 2020, ISSN 1337-8384, eISSN 2453-711X 
indispensable pour bien faire la différence entre les mots. Prenons un exemple simple :

Pour Noël, je dois acheter des timbres et un bon vin.

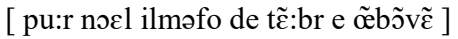

Pour bien saisir la valeur distinctive entre $\tilde{\varepsilon}$, œ̃ et $\tilde{\jmath}$ il nous faut énormément du temps et du travail pour atteindre le niveau attendu. Cela va de même pour la prononciation de la consomme " $\mathrm{r}$ », appelé aussi une fricative uvulaire, pour bien la prononcer, un apprenant étranger doit s'entrainer beaucoup jusqu'à ce que la prononciation soit suffisamment bonne.

En grammaire- nos étudiants ont beaucoup de travail à fournir avant d'obtenir le diplôme. Ils doivent non seulement savoir parler correctement la langue qu'ils apprennent mais aussi l'étudier dans sa forme grammaticale. Même si la morphologie fonctionnelle française est facile à comprendre son fonctionnement, pour un apprenant kosovar, il y a tellement de différences (car notre morphologie est fusionnelle) qu'un apprenant débutant doit savoir qu'il lui faudra passer un bon bout de temps à apprendre la grammaire française ; ce qui n'est pas une tâche à accomplir facilement. Nous devons emmener les étudiants à intégrer leurs apprentissages par la pratique d'une application (divers exercices et travaux pratiques, essais pour l'écriture, pour la lecture, réalisation d'une pièce de théâtre, etc.). Parmi les difficultés les plus accentuées, on en retient trois, même si l'on peut en trouver davantage sûrement :

- Les articles

- L'emploi de certains verbes et temps grammaticaux

- L'emploi des prépositions

Les articles - le français est une langue "avec article", l'albanais aussi, mais l'apprenant kosovar s'affronte avec une grande difficulté syntaxique au niveau de la distribution des articles dans les deux langues; nous rencontrons un article un français là où l'albanais ne autorise pas son équivalent et l'inversement. Les paires des exemples suivants illustrent bien ce phénomène ${ }^{7}$ :

(6) $\quad$ a. $\quad$ Le Paris est une belle ville.

b. $\quad$ Paris est une belle ville.

a. * Je suis dans voiture.

b. Je suis dans la voiture.

Une confusion d'article assez problématique apparait dans les débuts de l'apprentissage du français. À la différence du français, en albanais, l'article défini accompagne tout nom comme par exemple le mot Paris dans la phrase en (7) est muni obligatoirement de l'article défini en albanais, comme le montre la flexion- $i$ dans Paris-i është një qytet i bukur "Le Paris est une belle ville".

L'emploi de certains verbes et temps grammaticaux - La différence dans l'emploi de certaines temps montre bien l'effort qu'un apprenant albanais doit effectuer pour bien l'utiliser en français sans oublier les difficultés dans le choix de l'auxiliaire être ou avoir pour former les temps composés (J'ai vingt ans et non Je suis vingt ans/ Je

${ }^{7}$ L'astérisque $" * "$ indique que la phrase est mal formée. Ici, dans cette phrase, les données canoniques de la grammaire française nous imposent d'enlever l'article défini "le" et sans hésitation pour que la phrase soit syntaxiquement correcte. 
suis parti(e) et non J'ai parti). Concernant l'utilisation d'un temps grammatical, l'albanais montre une distribution morphosyntaxique plus large du «passé simple » qu'en français parce qu'il a une valeur temporelle du " passé au sens propre ». Le français préfère le passé composé (8a) :

$$
\begin{aligned}
& \text { a. J'ai acheté une voiture. } \\
& \text { b. J'achetai une voiture. }
\end{aligned}
$$

L'emploi des prépositions - Les apprenants albanophones doivent savoir que l'emploi de certaines prépositions françaises nécessite une étude très approfondie, voire une étude de plusieurs années pour bien connaître la distribution prépositionnelle française sous toutes ses formes. Autrement dit, une sélection d'une bonne préposition, dans chaque contexte, n'est pas un apprentissage facile ${ }^{8}$. Pour bien enseigner le choix des prépositions, nous avons utilisé, encore une fois, des outils numériques, il nous a fallu procéder étape par étape, en commençant par les phrases simples contenant des syntagmes prépositionnelles les plus simples et en expliquant leurs effets sémantiques associés. Par exemple, prenons le verbe parler, un verbe simple, certainement, mais qui demande toujours la préposition à en (9a), la préposition de en ( $9 b)$ et pas de préposition en $(9 \mathrm{c})$ :

$$
\begin{array}{ll}
\text { a. } & \text { Tous les jours, nous parlons à Tayna, à notre fils. } \\
\text { b. } & \text { Tous les jours, nous parlons de Tayna, de notre fils. } \\
\text { c. } & \text { Tous les jours, nous parlons politique, français, anglais. }
\end{array}
$$

Cette liste est loin d'être exhaustive ; elle sert uniquement à dégager les difficultés qu'un professeur peut les signaler lors de son expérience en classe de FLE, Cela peut être particulièrement difficile pour les débutants, les premières années d'études, ensuite, il progressera petit à petit ultérieurement lui-même. Pour convaincre les difficultés, heureusement, ils existent aujourd'hui plusieurs outils numériques, disponibles sur internet, uniquement pour les étudiants et ils en profitent parce que :

- $\quad$ Ils aperçoivent une progression en orthographe et évitent de faire des fautes d'orthographe.

- $\quad$ Ils arrivent à suivre le rythme des leçons de façon continue durant toutes leurs études.

- Ils n'sont pas perturbés par l'ambiance stressante de la classe. Avec ces nouvelles méthodes, ils peuvent regagner le goût de rattraper les leçons qu'ils ont perdues ou mal comprises.

- Les étudiants en difficulté n'ont plus peur de montrer leurs lacunes en classroom, le professeur n'y est pas pour le reprocher, ils peuvent donc reprendre un exercice autant de fois que nécessaire, pour une perfection en douceur.

- Ils peuvent également relire, revoir plusieurs fois les cours du prof mis en ligne afin qu'ils n'y aient pas de lacunes.

\footnotetext{
${ }^{8}$ Sur la distribution des prépositions en français, nous avons un très bon livre Est-ce à ou de ? I. Répertoire des verbes, adjectifs et locutions se construisant avec une préposition de Lasserre (1959). Dans ce livre, dans la partie Avant-propos, il se pose beaucoup de questions comme par exemple : " Pourquoi dit-on : " chercher à comprendre » et " essayer de comprendre » 》.
} 
- $\quad$ Ils renforcent les apprentissages, en pouvant se connecter partout grâce au classroom qui est vu comme un coach s'adaptant aux besoins de l'étudiant même si la base de l'enseignement reste bien l'université.

L'un des outils les plus utilisés lors des cours linguistiques en classe de FLE à Prishtina est Padlet et particulièrement pour les activités en salle de travaux pratiques. Étant attrayante et facile à utiliser et surtout efficace pour nos cours en classe. Une fois le compte ouvert sur Internet, nous avons la possibilité de rejoindre un Padlet (en général les étudiants rejoignent celui du professeur), d'en créer un nouveau (le cas du professeur) ou d'en créer plusieurs, et c'est exactement cette possibilité de «somme » ou réunion de plusieurs choses assemblés sur un seul mur faisant référence à un tableau classique qui que rend le cours plus fascinant et plus riche en information.

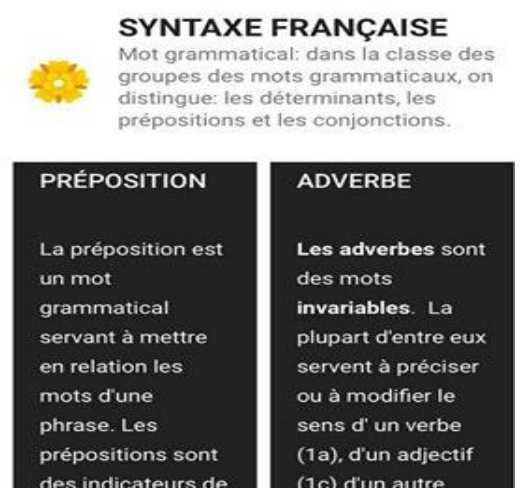

Figure 4 : Padlet crée pour un cours de langue française.

À l'Université de Prishtina, au cours de la dernière année universitaire, cette nouvelle méthode nous a aidé énormément; grâce au Padlet, les étudiants pouvaient s'entrainer assidûment à l'apprentissage du français (notamment sur l'emploi des prépositions, articles et conjugaison verbale), s'enregistrer et mettre en ligne, sur le mur Padlet. Ensuite, nous, les professeurs, nous corrigions les enregistrements ce qui nous servait comme un support d'évaluation. Ainsi chaque étudiant pouvait s'écouter, écouter les enregistrements de ses collègues et les corrigés de tous faits par le professeur. Nous avons fait ce que nous pensions être une bonne motivation pour que les étudiants puissent s'exprimer couramment en français et travailler plus régulièrement.

Le numérique aujourd'hui ne joue pas seulement un rôle de pédagogue virtuel, pour ne pas exagérer, il est vu également comme un outil psychologique pour détecter les points faibles de l'étudiant, tout en lui donnant la possibilité d'avoir plus de confiance en lui-même. Que du positif avec le numérique!

\section{Conclusions}

Notre article consiste à cerner comment les applications : Google classeroom, Padlet et Kahoot sont intégrées dans le bon processus de l'enseignement et de l'apprentissage du français à l'Université de Prishtina. Notre pratique enseignante a pris en compte les stratégies des apprenants avec des applications et nous avons constaté que les étudiants stockent plus durablement les informations comprises facilement. Sans outils numériques, aujourd'hui, il est difficile d'échapper à des mécompréhensions causant des difficultés importantes dans le processus d'enregistrement des données. La mémoire enregistre beaucoup mieux les éléments représentés simultanément par l'ouïe et la vue. Et, pour finir, selon notre expérience et 
les applications mentionnées dans ce travail, l'apprentissage dans une langue étranger nécessite assez d'entraînement sans quoi il serait insuffisamment efficace. Ce travail est fait en se basant sur des conseils méthodologiques et propose de mettre en place une stratégie ou astuce numérique pour mieux faciliter l'apprentissage en permettant aux apprenants du niveau universitaire d'avoir des bases solides littéraires et linguistiques. Nous avons tenté de montrer notre propre expérience d'enseignement avec nos objectifs pédagogiques et surtout comment une application doit se considérer comme un support pédagogique pour le développement professionnel. Toutefois, même si cet article est destiné aux étudiants voulant apprendre le français et ses réels atouts culturels, il peut servir aussi aux enseignants comme une nouvelle méthode de l'enseignement avec le numérique. Le processus d'apprentissage en classe de FLE cache tellement de surprises dans les premières années d'études pour les étudiants qui y sont indifférents au départ, mais que progressivement s'y habituent. Et pour devenir de futurs bons enseignants en FLE, posséder seulement une large connaissance des textes littéraires et linguistiques ne suffit plus, nous devons aussi savoir bien maitriser l'informatique, savoir trouver un moyen de nous faire connaître et échanger avec d'autres professionnels sur Internet, un outil inévitable dans le monde où l'on vit d'aujourd'hui. Dans un aspect pédagogique, ce type d'enseignement est très encourageant ; ce modèle s'adapte mieux pour chaque étudiant sans laisser de côté les éléments prescrits par le programme et la motivation est très valorisée par tous. En fait, nous sommes persuadées que toute réussite scolaire ou toute qualification reconnue de ses bonnes compétences dans une profession quelconque ne peut avoir pour origine que la méthode d'enseignement/apprentissage. Et c'est grâce à ces nouvelles méthodes pédagogiques numériques que nous voyons une transformation générale de l'éducation dans le développement global de nos sociétés.

\section{Bibliographic references}

ABDALLAH-PRETCEILLE, M. 2017. L'éducation interculturelle, 5e édition corrigée, Paris : PUF (Que sais-je?). ISBN 9782130792246.

ALI BOUACHA, A. 1978. La Pédagogie du français langue étrangère. Orientations théoriques, pratique dans la classe, Paris : Hachette. ISBN 10: 2010051890.

ASLIM-YETIS, V. 2009. Didactique des langues, évolution de la technologie et Internet. Sayfa, nr. 2, pp. 40-56. ISSN 1304-8880.

BIHOUÉ, P. - COLLIAUX, A. 2011.Enseigner différemment avec les tice. Paris: Groupe Eyrollles. ISBN : 978-2-212-55171-6.

CICUREL, F. 1991. Lectures interactives en langues étrangères, Paris : Hachette. ISBN 2010181522.

COIANIZ, A. 2003. « Méthodologie de l'enseignement du français et conceptions de l'homme », Tréma, 17 | 2000, pp. 3-26. ISSN : 1167-315X.

COLlES, L. 2013. Passage des frontières. Etudes de didactiques du français et de l'interculturel, Presses universitaires de Louvain : Louvain-la-Neuve (Belgique). ISBN 9782875582379.

CORDINA, D. - RAMBERT, J. - ODDOU, M. 2017. Pratiques et projets numériques en classe de FLE. Paris : CLE International. ISBN : 978-2-09-038230-3.

CUQ, J-P. 2003. Dictionnaire de didactique du français langue étrangère et seconde. Paris : CLE International - ASDIFLE. ISBN 2090339721.

DESMET, P. 2006. «L'enseignement/apprentissage des langues à l'ère du numérique: tendances récentes et défis ", Revue française de linguistique appliquée, 1 vol. XI , pp. 119-138. ISSN 1386-1204 Article disponible en ligne à l'adresse : https://www.cairn.inforevue-francaise-de-linguistique appliquee-2006-1-page119.htm.

XLinguae, Volume 13 Issue 1, January 2020, ISSN 1337-8384, eISSN 2453-711X 
DOMINE, G. 2017. Les TICE en classe, mode d'emploi. 3e édition. Paris : ESF éditeur : Cahiers pédagogiques. ISSN : 1158-4580.

LASSERRE, E. 1959. Est-ce à ou de ? I. Répertoire des verbes, adjectifs et locutions se construisant avec une préposition, Lausanne : Librairie Payot. ISBN-10: 2601012773.

LEBRUN, M. 2004. La formation des enseignants universitaires aux TIC: allier pédagogie et innovation. In Revue Internationale des Technologies en Pédagogie Universitaire. Revue patronnée par la Crepuq (Canada, Québec), 1, 1, pp.11-21.

GADENNE, J.-E. - SOTIRAKIS, B. 2003. J'enseigne avec l'Internet. Centre régional de documentation pédagogique de Bretagne. ISSN 1288-7676.

PREVOST, Ph. 2009. The acquisition of French. The development of inflecional morphology and syntax. L1 acquisition, bilingualism, and L2 acquisition. Amsterdam: John Benjamins. ISBN 9789027253125.

REBOULLET, A. 1973. L'enseignement de la civilisation française, Paris, Hachette. ISBN-10: 2010029917.

REBOULLET, A. 1971. Guide pédagogique pour le professeur de français langue étrangère, Paris: Hachette. ISBN : 2010004566.

Autres documents numériques, représentés par thème :

L'acquisition du lexique et l'apprentissage de la syntaxe, 2011. Available online: http://www.ac-grenoble.fr/ien.g1/IMG/pdf/lexique_syntaxe_LEM_DSR.pdf

L'acquisition du français à l'usage d'applications mobiles: un état de question. 2014. Available online:https://lib.ugent.be/fulltxt/RUG01/002/162/173/RUG01002162173_2014_0001_AC.pdf

Documents pour l'histoire du français langue étrangère ou seconde. Revue semestrielle. Available online: https://journals.openedition.org/dhfles/

La syntaxe en FLE [en ligne, page consultable et disponible sur le web].

Available online: https://www.accaen.fr/dsden50/circo/cherbourgouest/IMG/pdf/oral_ecrit_4_syntaxe.pdf

Words: 5927

Characters: 39803 (22,11 standard pages)

Porf. ass. Teutë Blakqori

Department of French Language and literature

Faculty of philology, University " Hasan Prishtina" of Prishtina

10000 Prishtina,

Kosovo

teuta.blakqori@uni-pr.edu 